\title{
Association of apolipoproteins $A 1$ and $B$ with type 2 diabetes and fasting blood glucose: a cross-sectional study
}

\author{
Liang $\mathrm{Gao}^{1+}$, Yaju Zhang ${ }^{2+}$, Xingmin Wang ${ }^{3^{*}}$ and Hongli Dong ${ }^{4^{*}}$ (D)
}

\begin{abstract}
Background: Apolipoprotein (Apo) may be associated with type 2 diabetes (T2D), however, little is known whether or not serum apolipoproteins are correlated with fasting blood glucose (FBG) and the prevalence of T2D in Chinese populations. In this study, we examined the association of serum ApoA1, ApoB, and the ratio of ApoB/ApoA1 (ApoB/A1 ratio) with T2D and FBG level, and compared apolipoprotein indicators in predicting T2D in Chinese adults.
\end{abstract}

Methods: A total of 1027 subjects were enrolled in this cross-sectional study. The association of ApoA1, ApoB, and ApoB/A1 ratio with T2D prevalence was determined using logistic regression models. Multivariate-analysis of covariance (ANCOVA) was performed for comparisons of the mean difference in FBG level.

Results: We found that $A p o B$ and $A p o B / A 1$ ratio were positively associated with T2D prevalence and FBG, while inverse association was noted between ApoA1 and T2D prevalence as well as FBG. Stratified analyses for sex, age, body mass index (BMI), smoking, and alcohol consumption showed no significant difference for the association of $A p o A 1, A p o B$, and ApoB/A1 ratio with the prevalence of T2D among subgroups (all $p$-interactions $>0.05$ ).

Nonetheless, ApoA1 poorly performed in predicting T2D as it provided an AUC value of 0.310 that was significantly lower than those observed for ApoB (AUC value: 0.631 ) and ApoB/A1 ratio (AUC value: 0.685 ). Finally, path analyses indicated that the association between $\mathrm{ApoB}$ and $\mathrm{T} 2 \mathrm{D}$ was mediated by BMI.

Conclusions: This study reveals the association of serum ApoA1, ApoB, and ApoB/A1 ratio with T2D and FBG in Chinese adults, suggesting that $A p o B$ and $A p o B / A 1$ ratio may be early indicators for predicting T2D. Prospective investigation in large cohort is needed.

Keywords: Apolipoprotein A1, Apolipoprotein B, Apolipoprotein B/A1 ratio, Type 2 diabetes, Fasting blood glucose

\footnotetext{
* Correspondence: xingmin-wang@ntu.edu.cn; dhljiyi@163.com

'Liang Gao and Yaju Zhang contributed equally to this work.

${ }^{3}$ Nantong Institute of Genetics and Reproductive Medicine, Affiliated

Maternity \& Child Health Care Hospital of Nantong University, Nantong

226018, Jiangsu Province, China

${ }^{4}$ Scientific Education Section, Affiliated Maternity \& Child Health Care Hospital of Nantong University, Nantong 226018, Jiangsu Province, China

Full list of author information is available at the end of the article
}

(C) The Author(s). 2021 Open Access This article is licensed under a Creative Commons Attribution 4.0 International License, which permits use, sharing, adaptation, distribution and reproduction in any medium or format, as long as you give appropriate credit to the original author(s) and the source, provide a link to the Creative Commons licence, and indicate if changes were made. The images or other third party material in this article are included in the article's Creative Commons. licence, unless indicated otherwise in a credit line to the material. If material is not included in the article's Creative Commons licence and your intended use is not permitted by statutory regulation or exceeds the permitted use, you will need to obtain permission directly from the copyright holder. To view a copy of this licence, visit http://creativecommons.org/licenses/by/4.0/ The Creative Commons Public Domain Dedication waiver (http://creativecommons.org/publicdomain/zero/1.0/) applies to the data made available in this article, unless otherwise stated in a credit line to the data. 


\section{Background}

Type 2 diabetes (T2D) is a major cause of death with an estimated 5 million death worldwide in 2019 [1]. The prevalence of T2D increased 16-fold over the past 3 decades and reached $10.9 \%$ in China $[1,2]$. Previous study indicated that lipoproteins exerted key effects on the pathogenesis of diabetes [3]. Therefore, the exploration of lipoprotein markers levels for T2D intervention is of high priority.

Traditional lipid concentrations are known to predict T2D, such as total cholesterol (TC), triglycerides (TG), high-density lipoprotein cholesterol (HDL-C) and lowdensity lipoprotein (LDL) particles [4]. There is growing interest in which lipid markers best assess diabetes risk [5-7]. Circulating apolipoproteins, which relate to the total number of lipoprotein particles, are superior to standard lipoprotein cholesterol measures for evaluating T2D risk $[8,9]$. Apolipoproteins exert important roles in the metabolism of lipoprotein and blood glucose $[10,11]$. Apolipoprotein A1 (ApoA1) and apolipoprotein B (ApoB) are two major types of apolipoprotein that had been well investigated. ApoA1 is the main lipoprotein associated with high-density lipoprotein cholesterol (HDL-C) [12]. $A p o B$ is a single molecule that is presented as in low-, intermediate-, and very low-density lipoproteins [13]. In vitro and animal studies revealed that ApoA1 and $A p o B$ modulated fasting blood glucose (FBG) level via improving insulin level [14-16]. Several studies have assessed the association of ApoA1, ApoB, and ApoB/A1 ratio with the FBG level showing inverse or null association with ApoA1 [17-19] but positive association with ApoB and ApoB/A1 ratio [11, 20, 21]. Notably, studies observed inverse and even positive association between ApoA1 and T2D presence [22-28], and positive association between T2D presence and ApoB as well as ApoB/A1 ratio [5, 23, 26, 2830]. These results suggested that ApoA1, ApoB, and $\mathrm{ApoB} / \mathrm{A} 1$ ratio may play important roles in maintaining FBG and T2D pathological process. Previous studies showed that Chinese adults had lower levels of ApoA, ApoB, and lipoprotein compared to Caucasians [31], however, little data is available for Chinese adults [24, 28] who differ from their Western counterparts in levels of lipoprotein [31]. Moreover, the different prevalence of obesity between Chinese and Western populations may have impact on the apolipoprotein-T2D association because body fat can affect the circulating glycometabolism [32, 33]. The apolipoprotein-T2D/FBG association in Chinese population remains unclear.

Obesity is an important risk factor for the pathogenesis of T2D [34]. Accumulating evidence support a significant association between circulating apolipoprotein level and obesity [35]. No study, however, investigated whether or not the association between apolipoproteins and T2D is mediated by obesity.
In this cross-sectional study, we investigated the association of ApoA1, ApoB, and ApoB/A1 ratio with T2D and FBG level, and analyzed the mediating effects of body mass index (BMI) for these associations, thus to find potential indicators for predicting T2D in Chinese population.

\section{Materials and methods Study subjects}

A total of 1027 subjects including 892 non-diabetic participants and 135 T2D patients in Nantong, China, during a period from November 2018 to December 2019 were enrolled in this cross-sectional study. All subjects were $>18$ years old with 590 men and 437 women. This study was approved by the Ethics Committee of Nantong Maternal and Child Healthcare Hospital (Y2018020). Written informed consent was obtained from all participants.

\section{Questionnaire interview and laboratory assays}

Structured questionnaire [36, 37] was conducted to collect detailed sociodemographic information including sex, age, marriage, alcohol consumption (current drinker or non-drinker), smoking status (current smoker or non-smoker), exercise (light, moderate, strenuous), hypoglycemic medication and lipid-lowering drugs use by face-to-face interviews. The participants' body height and weight were measured, and BMI was calculated. T2D was identified by FBG level $(\geq 7.0 \mathrm{mmol} / \mathrm{L})$, selfreport, and relevant treatments. Blood samples were obtained from the participants after a $10-\mathrm{h}$ fast. Serum TC and TG were measured using the Denka Seiken enzymatic colorimetric test (Tokyo, Japan). HDL-C and LDL-C were measured using commercial reagents (Beckman Coulter Inc., Brea, CA). The coefficients of variation (CVs) for TC, TG, HDL-C and LDL-C were 2.1, 1.5, 3.7 and $4.1 \%$, respectively. FBG was measured using a commercial kit (Roche Diagnostics GmbH, China). The CV of FBG was $2.7 \%$.

Serum ApoA1 and ApoB concentrations were determined according to standardized operation. Briefly, $3 \mu \mathrm{L}$ serum sample were mixed with $240 \mu \mathrm{L}$ reagent composed of phosphate buffer $(0.05 \mathrm{~mol} / \mathrm{L}, \mathrm{pH}=7.0)$ and polyethylene glycol $(30 \mathrm{~g} / \mathrm{L})$. The mixture was vortexed for $2 \mathrm{~min}$ and then incubated at $37^{\circ} \mathrm{C}$ for $5 \mathrm{~min}$. The lyophilized products containing ApoA1 or ApoB were used as calibrator. The polyethylene glycol-enhanced immunology turbidimetric assay was conducted to detect the concentrations of ApoA1 and ApoB in a 7600-010 automatic analyzer (Hitachi, Japan). The intra-assay CVs of ApoA1 and $\mathrm{ApoB}$ were 3.2 and 4.9\%, respectively. The inter-assay CVs of ApoA1 and ApoB were 4.7 and $5.1 \%$, respectively. ApoB/A1 ratio was calculated. 


\section{Statistical analyses}

The baseline characteristics of the participants were described by means with standard deviations (SD) for the continuous variables and frequencies (percentages) for the categorical variables stratified by T2D status (Yes/ No). Student's t-test or the Mann-Whitney U test was used to compare the continuous variables and Chisquare test for the comparisons of the categorical variables. The subjects were categorized into quartiles according to ApoA1, ApoB, and ApoB/A1 ratio, respectively. Logistic regression model was used to estimate the association of $\mathrm{ApoA} 1, \mathrm{ApoB}$, and $\mathrm{ApoB} / \mathrm{A} 1$ ratio with the prevalence of T2D. Odds ratios (ORs) and 95\% confidence intervals (CIs) of the T2D for the 2nd 4th quartiles of apolipoprotein concentrations were computed, with the lowest quartile defined as the reference. Multivariate-analyses of covariance (ANCOVAs) were used to compare the mean differences of FBG level and test trends in quartiles of apolipoproteins. The Bonferroni test was performed for pair-wise comparisons among quartiles. In Model 1, age was adjusted. In Model 2, sex, age, marriage, alcohol consumption, smoking status, exercise, and hypoglycemic drug use were adjusted. In Model 3, lipid-lowering drugs use was further adjusted. Stratified analyses were performed by sex (Men vs. Women), age ( $\leq 60$ vs. $>60$ years), BMI ( $\leq 24$ vs. $>$ $24 \mathrm{~kg} / \mathrm{m}^{2}$ ), smoking (Yes vs. No) and drinking (Yes vs. No). Multiplicative interactions were assessed through likelihood ratio test. The receiver-operating characteristic (ROC) curves were drawn and values of area under curve (AUC) were estimated. Path analyses were conducted to assess the mediating effects of BMI on apolipoproteins-T2D association [38]. Two parts of path analyses were performed: one related to the association of apolipoproteins with BMI, the other related to the association of BMI with T2D. Standardized regression coefficients were obtained to evaluate correlation in each path. Two-tailed $p<0.05$ was considered statistically significant. The analyses were performed using SPSS Statistics 21.0 (IBM SPSS Statistics, Inc., Armonk, NY, USA). Path analyses were conducted using SPSS AMOS21.0 (IBM Corporation, Armonk, NY, USA).

\section{Results}

\section{Characteristics of the study subjects}

As shown in Table 1, the mean age of the T2D subjects and non-T2D participants was 63.6 and 59.8 years, respectively. Participants with T2D exhibited significantly higher BMI $\left(24.6 \mathrm{~kg} / \mathrm{m}^{2}\right.$ vs. $\left.23.9 \mathrm{~kg} / \mathrm{m}^{2}\right)$, FBG level $(8.95$ $\mathrm{mmol} / \mathrm{L}$ vs. $5.36 \mathrm{mmol} / \mathrm{L})$, TC $(5.79 \mathrm{mmol} / \mathrm{L}$ vs. 5.58 $\mathrm{mmol} / \mathrm{L}), \quad$ TG $(1.92 \mathrm{mmol} / \mathrm{L}$ vs. $1.58 \mathrm{mmol} / \mathrm{L})$, ApoB $(1.29 \mathrm{~g} / \mathrm{L}$ vs. $1.14 \mathrm{~g} / \mathrm{L})$, and $\mathrm{ApoB} / \mathrm{A} 1$ ratio $(1.88$ vs. 1.08$)$ and lower HDL-C $(1.24 \mathrm{mmol} / \mathrm{L}$ vs. $1.34 \mathrm{mmol} / \mathrm{L})$ and
ApoA1 $(0.85 \mathrm{~g} / \mathrm{L}$ vs. $1.21 \mathrm{~g} / \mathrm{L})$ compared to non-diabetic participants (all $p<0.05$ ).

\section{Association of ApoA1, ApoB, and ApoB/A1 ratio with T2D prevalence}

We initially analyzed whether or not serum ApoA1, $A p o B$, and $A p o B / A 1$ ratio were associated with T2D prevalence and found a trend that serum ApoA1 was inversely associated with $\mathrm{T} 2 \mathrm{D}$ prevalence. In contrast, $\mathrm{ApoB}$ and $\mathrm{ApoB} / \mathrm{A} 1$ ratio were positively associated with T2D prevalence in three models (Table 2). In Model 1 with adjustment for age, the ORs $(95 \% \mathrm{CI})$ in the fourth quartile were $0.29(0.18,0.46)$ for inverse association of serum ApoA1, $3.09(1.71,5.55)$ for positive association of ApoB, and $5.12(2.91,9.02)$ for positive association of ApoB/A1 ratio compared with the first quartile, respectively. In Model 2 with adjustment for other potential covariates, inverse associations for ApoA1 were found with ORs $(95 \% \mathrm{CI})$ of $0.15(0.07,0.33)$ in the second quartile, and of $0.37(0.20,0.68)$ in the third quartile compared with the first quartile, respectively. In contrast, positive associations for $A p o B$ and $A p o B / A 1$ ratio were found with ORs $(95 \%$ CI) of $3.52(1.67,7.41)$ and $2.53(1.35$, 4.73 ) in the fourth quartile compared to first quartile, respectively ( $p$-trend: $<0.001-0.023)$. After further adjusting for lipid-lowering drugs use in Model 3, the prevalence of T2D in the second and third quartiles of ApoA1 decreased $85 \%(\mathrm{OR}=0.15,95 \% \mathrm{CI}: 0.07,0.34)$ and $63 \%(\mathrm{OR}=0.37,95 \% \mathrm{CI}: 0.20,0.68)$ compared to the first quartile, respectively. The multivariable-adjusted ORs $(95 \% \mathrm{CI})$ of the T2D presence for the fourth quartile were 3.38 (1.60, 7.14) for ApoB and $2.39(1.27,4.52)$ for $\mathrm{ApoB} / \mathrm{A} 1$ ratio compared to first quartile, respectively ( $p$-trend: <0.001-0.036). In stratified analyses by sex, age, BMI, smoking, and drinking, the association of ApoA1, ApoB, and ApoB/A1 ratio with T2D prevalence was not significantly different among subgroups (all $p$ interactions $>0.05$, Table 3 and Supplementary Table 1).

\section{Association of lipid parameters with T2D prevalence}

Among lipid parameters, HDL-C was inversely associated with T2D prevalence, but positive associations of TG and LDL-C with T2D prevalence were detected across the three models. Null association was found between TC and T2D prevalence in multivariate analysis (Supplementary Table 2).

\section{Association of $A p o A 1, A p o B$, and $A p o B / A 1$ ratio with $F B G$ level}

In three Models, serum ApoA1 exhibited an inverse association with $F B G$, while $A p o B$ and $A p o B / A 1$ ratio showed positive association with FBG (Table 4). With adjustment for age in Model 1, lower FBG level was associated with higher ApoA1 concentration. In contrast, 
Table 1 Characteristics of the study participants

\begin{tabular}{|c|c|c|c|}
\hline Variables & T2D $(n=135)$ & Non-T2D $(n=892)$ & $p$ \\
\hline Age, years & $63.6 \pm 13.5$ & $59.8 \pm 10.5$ & $<0.001$ \\
\hline Female, n (\%) & $63(41.93)$ & $374(46.67)$ & 0.299 \\
\hline $\mathrm{BMI}, \mathrm{kg} / \mathrm{m}^{2}$ & $24.6 \pm 3.3$ & $23.9 \pm 5.5$ & 0.047 \\
\hline Marriage, n (\%) & & & 0.584 \\
\hline Married & $123(91.1)$ & $818(91.7)$ & \\
\hline Unmarried & $1(0.7)$ & $15(1.7)$ & \\
\hline Divorce/Widowed & $11(8.2)$ & $59(6.6)$ & \\
\hline Education level, n (\%) & & & 0.033 \\
\hline Junior high school & $100(74.1)$ & $605(67.8)$ & \\
\hline High school & $26(19.3)$ & $153(17.2)$ & \\
\hline College degree or above & $9(6.6)$ & $134(15.0)$ & \\
\hline Smoker, n (\%) & $25(18.5)$ & $189(21.2)$ & 0.856 \\
\hline Alcohol drinker, n (\%) & $57(42.2)$ & $384(43.0)$ & 0.477 \\
\hline Hypoglycemic drugs use, n (\%) & $43(31.9)$ & $0(0.0)$ & $<0.001$ \\
\hline Lipid-lowering drugs use, n (\%) & $12(8.1)$ & $48(5.4)$ & 0.105 \\
\hline Exercise, n (\%) & & & 0.455 \\
\hline Sitting & $58(43.0)$ & $343(38.5)$ & \\
\hline Light & $57(42.2)$ & $366(41.0)$ & \\
\hline Moderate & $15(11.1)$ & $141(15.8)$ & \\
\hline Strenuous & $5(3.7)$ & $42(4.7)$ & \\
\hline $\mathrm{FBG}, \mathrm{mmol} / \mathrm{L}$ & $8.95 \pm 1.65$ & $5.36 \pm 0.66$ & $<0.001$ \\
\hline $\mathrm{TC}, \mathrm{mmol} / \mathrm{L}$ & $5.79 \pm 1.09$ & $5.58 \pm 1.16$ & 0.043 \\
\hline $\mathrm{TG}, \mathrm{mmol} / \mathrm{L}$ & $1.92 \pm 1.10$ & $1.58 \pm 1.06$ & $<0.001$ \\
\hline $\mathrm{HDL}-\mathrm{C}, \mathrm{mmol} / \mathrm{L}$ & $1.24 \pm 0.31$ & $1.34 \pm 0.34$ & 0.002 \\
\hline $\mathrm{LDL}-\mathrm{C}, \mathrm{mmol} / \mathrm{L}$ & $3.58 \pm 0.89$ & $3.54 \pm 0.96$ & 0.620 \\
\hline \multicolumn{4}{|l|}{ Apolipoproteins, g/L } \\
\hline ApoA1 & $0.85 \pm 0.58$ & $1.21 \pm 0.40$ & $<0.001$ \\
\hline ApoB & $1.29 \pm 0.34$ & $1.14 \pm 0.33$ & $<0.001$ \\
\hline ApoB/A1 ratio & $1.88 \pm 1.30$ & $1.08 \pm 0.64$ & $<0.001$ \\
\hline
\end{tabular}

$B M I$ body mass index, FBG fasting blood glucose, Apo apolipoprotein, $T C$ total cholesterol, TG triglyceride, HDL-C high-density lipoprotein cholesterol, $L D L-C$ lowdensity lipoprotein cholesterol, T2D type 2 diabetes

increased FBG level was detected in subjects with higher ApoB level and ApoB/A1 ratio. With adjustment for the other potential covariates in Model 2, intermediate ApoA1 concentration was negatively associated with FBG level (Q2 vs. Q1 = 5.574 mmol/L vs. $6.067 \mathrm{mmol} / \mathrm{L}$; Q3 vs. Q2 $=5.881 \mathrm{mmol} / \mathrm{L}$ vs. $5.574 \mathrm{mmol} / \mathrm{L}$ ). In contrast, $\mathrm{ApoB}$ and $\mathrm{ApoB} / \mathrm{A} 1$ ratio were positively associated with $\mathrm{FBG}$ level $(\mathrm{Q} 4$ vs. $\mathrm{Q} 1=5.910 \mathrm{mmol} / \mathrm{L}$ vs. $5.728 \mathrm{mmol} / \mathrm{L}$ for $\mathrm{ApoB}$; Q3 vs. Q2 $=5.679 \mathrm{mmol} / \mathrm{L}$ vs. $5.972 \mathrm{mmol} / \mathrm{L}$ for ApoB/A1 ratio). After further adjusting for lipid-lowering drugs use in Model 3, higher ApoA1 concentration were associated with significantly lower FBG level (Q2 vs. Q1 $=5.575 \mathrm{mmol} / \mathrm{L}$ vs. 6.067 $\mathrm{mmol} / \mathrm{L}$ ). In contrast, positive association between ApoB and $A p o B / A 1$ ratio and $F B G$ level were shown. The corresponding FBG values were $5.908 \mathrm{mmol} / \mathrm{L}$ (vs. 5.730 $\mathrm{mmol} / \mathrm{L}$ ) for $\mathrm{ApoB}$ in the fourth quartile compared with the first quartile, and $5.678 \mathrm{mmol} / \mathrm{L}$ (vs. $5.971 \mathrm{mmol} / \mathrm{L}$ ) for $\mathrm{ApoB} / \mathrm{A} 1$ ratio in the third quartile compared with the second quartile.

\section{Performance of apolipoprotein indicators}

AUC was used to evaluate the performance of ApoA1, $\mathrm{ApoB}$, and $\mathrm{ApoB} / \mathrm{A} 1$ ratio for predicting T2D (All $p<$ 0.001 ). As shown in Table 5 , ApoA1 poorly performed at predicting T2D with an AUC value of 0.310 that was significantly smaller than AUCs observed from $A p o B$ (AUC value: 0.631 ) and $A p o B / A 1$ ratio (AUC value: 0.685 ). The corresponding ROC curves were shown in Supplementary Fig. 1, 2 and 3. 
Table 2 The associations of ApoA1, ApoB levels and ApoB/A1 ratio with T2D prevalence

\begin{tabular}{|c|c|c|c|c|c|}
\hline \multirow[t]{2}{*}{ Variables } & \multicolumn{4}{|c|}{ Quartiles by Apolipoproteins } & \multirow[t]{2}{*}{$p$} \\
\hline & $\overline{\text { Q1 }}$ & Q2 & Q3 & Q4 & \\
\hline \multicolumn{6}{|l|}{ ApoA1, g/L } \\
\hline Mean (SD) & $0.59(0.20)$ & $0.99(0.10)$ & $1.34(0.10)$ & $1.73(0.16)$ & \\
\hline Case/N & $76 / 255$ & $10 / 255$ & $20 / 257$ & $29 / 258$ & \\
\hline \multicolumn{6}{|l|}{ OR $(95 \% \mathrm{Cl})$} \\
\hline Model 1 & 1 & $0.09(0.05,0.18)^{* *}$ & $0.20(0.12,0.35)^{* *}$ & $0.29(0.18,0.46)^{* *}$ & $<0.001$ \\
\hline Model 2 & 1 & $0.15(0.07,0.33)^{* *}$ & $0.37(0.20,0.68)^{* *}$ & $0.60(0.35,1.02)$ & 0.232 \\
\hline Model 3 & 1 & $0.15(0.07,0.34)^{* *}$ & $0.37(0.20,0.68)^{* *}$ & $0.60(0.35,1.03)$ & 0.232 \\
\hline \multicolumn{6}{|l|}{ ApoB, g/L } \\
\hline Mean (SD) & $0.77(0.14)$ & $1.03(0.06)$ & $1.23(0.06)$ & $1.60(0.25)$ & \\
\hline Case/N & $17 / 262$ & $25 / 252$ & $46 / 260$ & $47 / 253$ & \\
\hline \multicolumn{6}{|l|}{ OR $(95 \% \mathrm{Cl})$} \\
\hline Model 1 & 1 & $1.53(0.80,2.91)$ & $2.91(1.62,5.24)^{* *}$ & $3.09(1.71,5.55)^{* *}$ & $<0.001$ \\
\hline Model 2 & 1 & $1.68(0.75,3.79)$ & $3.59(1.72,7.49)^{* *}$ & $3.52(1.67,7.41)^{* * *}$ & $<0.001$ \\
\hline Model 3 & 1 & $1.65(0.73,3.73)$ & $3.49(1.67,7.31)^{* *}$ & $3.38(1.60,7.14)^{* *}$ & $<0.001$ \\
\hline \multicolumn{6}{|l|}{ ApoB/A1 ratio } \\
\hline Mean (SD) & $0.58(0.08)$ & $0.79(0.07)$ & $1.10(0.13)$ & $2.26(0.95)$ & \\
\hline Case/N & $17 / 256$ & $31 / 257$ & $16 / 258$ & $71 / 256$ & \\
\hline \multicolumn{6}{|l|}{ OR (95\% Cl) } \\
\hline Model 1 & 1 & $1.82(0.98,3.38)$ & $0.84(0.41,1.71)$ & $5.12(2.91,9.02)^{* *}$ & $<0.001$ \\
\hline Model 2 & 1 & $1.56(0.82,2.97)$ & $0.75(0.36,1.58)$ & $2.53(1.35,4.73)^{* *}$ & 0.023 \\
\hline Model 3 & 1 & $1.50(0.78,2.87)$ & $0.72(0.34,1.52)$ & $2.39(1.27,4.52)^{*}$ & 0.036 \\
\hline
\end{tabular}

Model 1 was adjusted for age. Model 2 was adjusted for sex, age, BMI, education, marriage, exercise, cigarette smoking, alcohol consumption and hypoglycemic drugs use. Model 3 further adjusted for lipid-lowering drugs use

Apo apolipoprotein, $S D$ standard deviation, $O R$ odds ratio, 95\% Cl 95\% confidence interval, $Q$ quartile, T2D type 2 diabetes

* $p<0.05$ compared with Q1

${ }^{* *} p<0.001$ compared with Q1

Table 3 The associations of ApoA1, ApoB levels and ApoB/A1 ratio with T2D prevalence by sex (OR, 95\% Cl)

\begin{tabular}{|c|c|c|c|c|c|}
\hline \multirow[t]{2}{*}{ Variables } & \multicolumn{4}{|c|}{ Quartiles by Apolipoproteins } & \multirow{2}{*}{$\begin{array}{l}p \text { - } \\
\text { interaction }\end{array}$} \\
\hline & Q1 & Q2 & Q3 & Q4 & \\
\hline ApoA1 & & & & & 0.136 \\
\hline Men & 1 & $0.32(0.12,0.86)^{*}$ & $0.74(0.33,1.65)$ & $0.98(0.45,2.11)$ & \\
\hline Women & 1 & $0.05(0.01,0.22)^{* *}$ & $0.14(0.05,0.40)^{* *}$ & $0.38(0.17,0.86)^{*}$ & \\
\hline ApoB & & & & & 0.727 \\
\hline Men & 1 & $1.24(0.44,3.51)$ & $2.44(0.97,6.16)$ & $2.59(1.02,6.58)^{*}$ & \\
\hline Women & 1 & $2.74(0.69,10.82)$ & $5.56(1.53,20.25)^{*}$ & $4.46(1.19,16.70)^{*}$ & \\
\hline ApoB/A1 ratio & & & & & 0.792 \\
\hline Men & 1 & $1.42(0.59,3.44)$ & $0.92(0.36,2.38)$ & $2.09(0.87,5.01)$ & \\
\hline Women & 1 & $1.18(0.44,3.19)$ & $0.32(0.09,1.17)$ & $2.53(0.95,6.74)$ & \\
\hline
\end{tabular}

Age, BMl, education, marriage, exercise, cigarette smoking, alcohol consumption, hypoglycemic drugs use, and lipid-lowering drugs use were adjusted Apo apolipoprotein, $O R$ odds ratio, $95 \% C l 95 \%$ confidence interval, $Q$ quartile, $T 2 D$ type 2 diabetes ${ }^{*} p<0.05$ compared with Q1

${ }^{* *} p<0.001$ compared with Q1 
Table 4 Mean FBG level according to quartiles of ApoA1, ApoB levels and ApoB/A1 ratio

\begin{tabular}{|c|c|c|c|c|c|c|}
\hline \multirow[t]{2}{*}{ Variables } & \multicolumn{4}{|c|}{ Quartiles by Apolipoproteins } & \multirow[t]{2}{*}{$p$-Diff } & \multirow{2}{*}{$\begin{array}{l}p \text { - } \\
\text { Trend }\end{array}$} \\
\hline & $\overline{\mathrm{Q} 1}$ & Q2 & Q3 & Q4 & & \\
\hline \multicolumn{7}{|l|}{ ApoA1 } \\
\hline$n$ & 255 & 257 & 257 & 258 & & \\
\hline \multicolumn{7}{|l|}{$\mathrm{FBG}, \mathrm{mmol} / \mathrm{L}$} \\
\hline Model 1 & $6.891 \pm 0.113$ & $5.316 \pm 0.113^{* *}$ & $5.636 \pm 0.113^{* *}$ & $5.802 \pm 0.113^{* * \#}$ & $<0.001$ & $<0.001$ \\
\hline Model 2 & $6.067 \pm 0.078$ & $5.574 \pm 0.074^{* *}$ & $5.881 \pm 0.074^{\#}$ & $6.117 \pm 0.074^{\# \#}$ & $<0.001$ & 0.184 \\
\hline Model 3 & $6.067 \pm 0.078$ & $5.575 \pm 0.074^{* *}$ & $5.880 \pm 0.075^{\#}$ & $6.117 \pm 0.074^{\# \#}$ & $<0.001$ & 0.184 \\
\hline \multicolumn{7}{|l|}{ ApoB } \\
\hline$n$ & 262 & 252 & 260 & 253 & & \\
\hline \multicolumn{7}{|l|}{$\mathrm{FBG}, \mathrm{mmol} / \mathrm{L}$} \\
\hline Model 1 & $5.637 \pm 0.117$ & $5.842 \pm 0.119$ & $6.136 \pm 0.117^{*}$ & $6.026 \pm 0.119$ & 0.017 & 0.006 \\
\hline Model 2 & $5.728 \pm 0.074$ & $5.914 \pm 0.075$ & $6.087 \pm 0.074^{*}$ & $5.910 \pm 0.075$ & 0.009 & 0.034 \\
\hline Model 3 & $5.730 \pm 0.075$ & $5.914 \pm 0.075$ & $6.087 \pm 0.074^{*}$ & $5.908 \pm 0.076$ & 0.010 & 0.038 \\
\hline \multicolumn{7}{|l|}{ ApoB/A1 ratio } \\
\hline $\mathrm{n}$ & 256 & 257 & 258 & 256 & & \\
\hline \multicolumn{7}{|l|}{$\mathrm{FBG}, \mathrm{mmol} / \mathrm{L}$} \\
\hline Model 1 & $5.622 \pm 0.115$ & $5.787 \pm 0.114$ & $5.419 \pm 0.114$ & $6.814 \pm 0.114^{* * \# \uparrow}$ & $<0.001$ & $<0.001$ \\
\hline Model 2 & $5.906 \pm 0.076$ & $5.972 \pm 0.074$ & $5.679 \pm 0.075^{\#}$ & $6.083 \pm 0.077^{\llbracket}$ & 0.002 & 0.499 \\
\hline Model 3 & $5.910 \pm 0.077$ & $5.971 \pm 0.074$ & $5.678 \pm 0.075^{\#}$ & $6.080 \pm 0.078^{\natural}$ & 0.002 & 0.533 \\
\hline
\end{tabular}

Model 1 was adjusted for age. Model 2 was adjusted for sex, age, BMI, education, marriage, exercise, cigarette smoking, alcohol consumption and hypoglycemic drugs use. Model 3 further adjusted for lipid-lowering drugs use

Apo apolipoprotein, $F B G$ fasting blood glucose, $Q$ quartile. $p$-Diff: Multiple comparison among quartiles

* $p<0.05$ compared with Q1

${ }^{* *} p<0.001$ compared with Q1

$\# p<0.05$ compared with Q2

$\# p<0.001$ compared with Q2

" $p<0.05$ compared with Q3

\section{Path analyses}

Path analyses indicated that ApoB-T2D prevalence association was mediated by BMI (Fig. 1). BMI was positively correlated to increased serum ApoB level $(r=0.069, p<$ $0.05)$ and $T 2 D$ prevalence $(r=0.073, p<0.05)$. Serum ApoA1 and $\mathrm{ApoB} / \mathrm{A} 1$ ratio had no direct effect on BMI (all $p>0.05$ ). No mediating effect of BMI on the association of ApoA1-T2D and ApoB/A1-T2D was found.

\section{Discussion}

In this community-based cross-sectional study, we estimated the association of serum levels of ApoA1 and $A p o B$ as well as the ratio of $\mathrm{ApoB} / \mathrm{A} 1$ with $\mathrm{T} 2 \mathrm{D}$ prevalence and FBG level. We found that serum ApoA1 was favorably associated with a decreased prevalence of T2D, whereas
$\mathrm{ApoB}$ and $\mathrm{ApoB} / \mathrm{A} 1$ ratio were positively associated with T2D prevalence. Consistent with these findings, we observed an inverse association of serum ApoA1 with FBG, and positive association of $A p o B$ and $A p o B / A 1$ ratio with FBG. Moreover, $\mathrm{ApoB}$ and $\mathrm{ApoB} / \mathrm{A} 1$ ratio performed better than ApoA1 in predicting T2D prevalence in a Chinese population.

ApoA1, a major protein component of HDL with antiinflammatory and immunomodulatory effect [12, 39], is found in significant amounts on chylomicron remnants [40]. The protective effect of HDL is due to lipoprotein(a) particles, especially ApoA1 [41]. Our results demonstrated that both ApoA1 and HDL-C were inversely associated with the T2D prevalence and an intermediate ApoA1 concentration showed a lower T2D prevalence compared with

Table 5 The ROC curves parameters for ApoA1, ApoB levels and ApoB/A1 ratio in predicting T2D

\begin{tabular}{llllll}
\hline Variables & AUC & $\mathbf{9 5 \%}$ Cl & Sensitivity (\%) & Specificity (\%) & $\boldsymbol{p}$ value \\
\hline ApoA1 & 0.310 & $0.249,0.371$ & 0.156 & 0.846 & 0.611 \\
ApoB & 0.631 & $0.584,0.679$ & 0.622 & 0.001 \\
ApoB/A1 ratio & 0.685 & $0.632,0.739$ & 0.489 & 0.861 & $<.001$ \\
\hline
\end{tabular}

Apo apolipoprotein, AUC area under curve, $R O C$ receivers operator characteristic, $T 2 D$ type 2 diabetes, $95 \% C l 95 \%$ confidence interval 

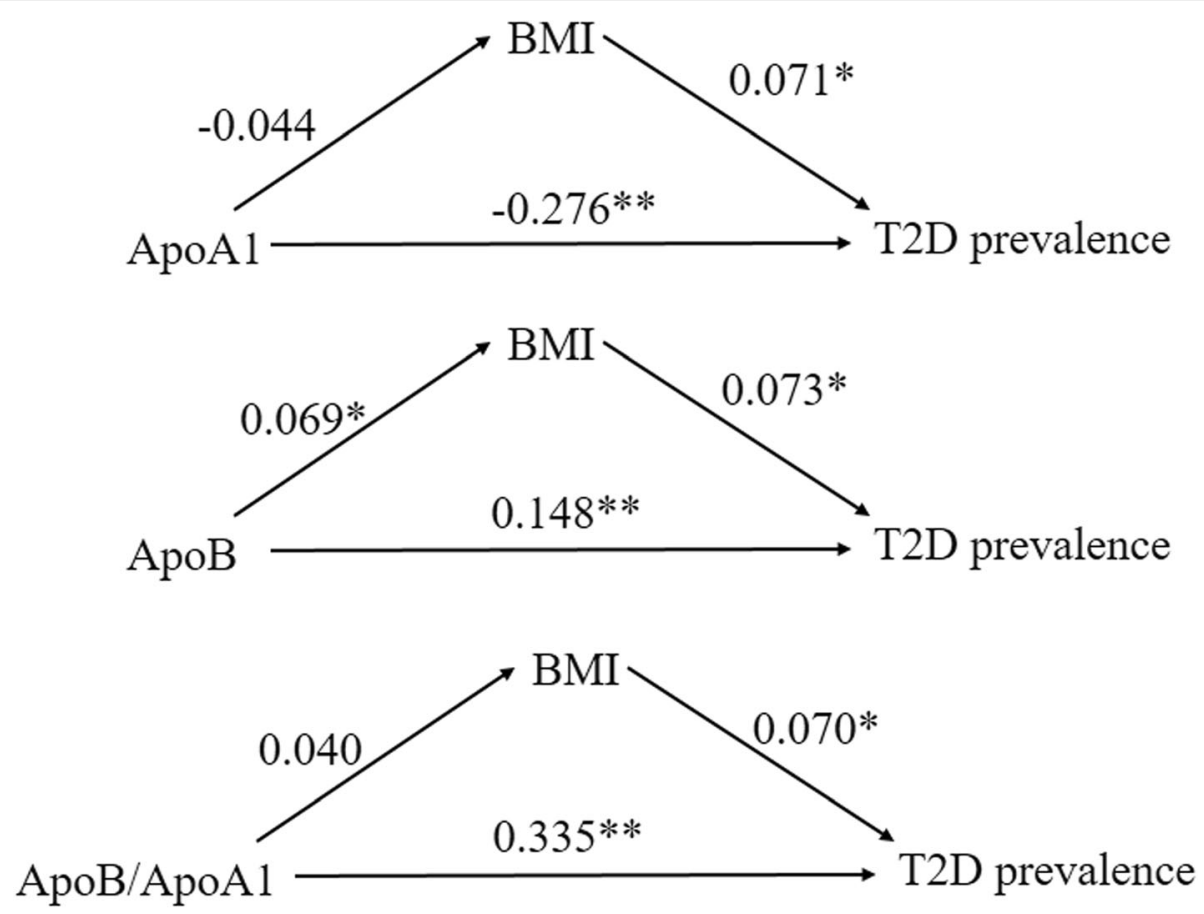

Fig. 1 Path analyses of associations of ApoA1, ApoB levels, ApoB/A1 ratio, and mediators (BMI) with the prevalence of T2D in Chinese adults, respectively. Apo, apolipoprotein; BMl, body mass index; T2D, Type 2 diabetes. * $p<0.05$; ** $p<0.001$

HDL-C. Similarly, ApoA1 has been observed to be equivalent or better than HDL-C as a risk marker for cardiovascular or atherosclerosis events [42, 43]. Previous studies reported that ApoA1 had cardio- and bone mass-protective roles [39, 44]. Although ApoA1 can modulate FBG, the association of ApoA1 with T2D prevalence and FBG level in Chinese populations is ill-characterized. Based on data from 1514 males participated in the ATTICA 2001-2012 surveys, the 10 -year T2D risk decreased $1.02 \%$ with every 1 $\mathrm{mg} / \mathrm{dL}$ increase of apoA1 [22]. FINRISK97 cohort consisted of 7827 middle aged persons found that ApoA were significantly associated with incident diabetes in Finland [45]. Wu et al. observed that low level of ApoA1 was independently associated with T2D (OR: 0.51, 95\% CI: 0.33, 0.76) [24]. TwSHHH indicated the inverse association between ApoA1 and incident T2D among 4223 adults in Taiwan, Chinese (hazard ratio [HR]: 0.59, 95\% CI: 0.38, 0.92) [28]. But fasting plasma glucose level was adjusted in multivariate analysis of this prospective study, which might be more likely to obtain inverse associations. Similar results were also reported by other groups $[23,25,26]$. Consistent with these findings, we observed a beneficial effect of ApoA1 on T2D presence. In contrast, null and even positive association was observed in western population [22, 27, 46]. Mellor et al. reported that serum ApoA1 was not associated with the risk of diabetes in a prospective cohort composed of 759 females in Greek population [22]. In a 13.5 years prospective study with 110 incident T2D and 741 non-T2D subjects within the Rotterdam Study, Brahimaj et al. [46] found null association between serum ApoA1 and T2D risk in the Dutch population. Onat et al. even found the positive association of serum ApoA1 with diabetes in Turkish population (OR: 1.85, 95\% CI: 1.03, 3.32) [27]. In addition, several studies assessed the association of ApoA1 with FBG level showing inconsistent results (inverse or null association) [18, 19, 47]. Similar to our results, previous studies found plasma glucose levels were negatively correlated to ApoA1 in Chinese and Dutch population (r: -0.1182 and -0.013) $[18,46]$. The heterogeneity among different studies can be caused by different mechanisms. Significant results were more likely to be obtained in cross-sectional study (vs. prospective study [22]). The lower ApoA1 concentration that was found in The Rotterdam Study $(7.4 \mu \mathrm{mol} / \mathrm{L})$ [46] than in the present study $(39.0 \mu \mathrm{mol} / \mathrm{L})$ might provide a potential explanation for the null findings of the prior one study. ApoA1 might turn to enhance risk of diabetes due to dysfunctional adiponect in prevailing among Turks (vs. this study) [27]. Moreover, favorable association is readily to be found in large sample size, non-obesity population, and Asian population as observed in the present study and previously reported. Although we were unable to characterize the shape of the exposure-outcome relation due to the limited number of samples, our results implied that an intermediate ApoA1 level was important in FBG regulation. Large prospective study is needed to confirm this speculation. 
A series of studies have shown the association of serum $A p o B$ and $A p o B / A 1$ ratio with T2D prevalence and FBG level [5, 7, 20-23, 27, 28, 30, 48]. A large multiethnic cohort study of kidney transplant recipients found that serum $\mathrm{ApoB}$ and $\mathrm{ApoB} / \mathrm{A} 1$ ratio were positively associated with post-transplantation diabetes mellitus with an OR of 12.2 (95\%CI: 3.13, 47.5) for ApoB and OR of 8.25 (95\%CI: 1.72, 39.5) for $\mathrm{ApoB} / \mathrm{A} 1$ ratio, respectively [23]. A retrospective longitudinal study in Korea also observed that $A p o B$ level and $A p o B / A 1$ ratio were positively associated with $\mathrm{T} 2 \mathrm{D}$, the $\mathrm{OR}$ values were 1.262 (95\%CI: 1.107, 1.438) for ApoB and 1.292 (95\%CI: 1.137, 1.468) for $\mathrm{ApoB} / \mathrm{A} 1$ ratio, respectively [5]. TwSHHH study also pointed out the risk of T2D associated with serum levels of $\mathrm{ApoB}$ and $\mathrm{ApoB} / \mathrm{A} 1$ ratio among adults in Taiwan, China (OR ${ }_{\mathrm{ApoB}}: 2.21,95 \% \mathrm{CI}_{\mathrm{ApoB}}: 1.12$, 4.16; $\mathrm{OR}_{\mathrm{ApoB} / \mathrm{A} 1}$ : 2.46, 95\% CI $\left.\mathrm{ApoB} / \mathrm{A} 1_{1}: 1.32,4.58\right)$ [28]. But fasting plasma glucose level was adjusted in this prospective study. In addition, a cross-sectional study with 70,063 subjects found a positive association of ApoB/A1 ratio with T2D (OR: 1.31, 95\%CI: 1.17, 1.46) [30]. Similar to these findings, the present study found positive association of $A p o B$ level and $A p o B / A 1$ ratio with T2D prevalence. Our study also confirmed the positive association of $\mathrm{ApoB}$ and $\mathrm{ApoB} / \mathrm{A} 1$ ratio with FBG level as previously reported $[20,21]$. However, the Atherosclerosis Risk in Communities (ARIC) study of 9026 American populations reported the null effects of $A p o B$ and $A p o B /$ A1 ratio on diabetes [7]. Analogous findings were seen in Turkish population [27], Australian women with previous gestational diabetes mellitus [48] and Greek population [22]. The inconsistent results of the present and previous studies might be attributable to some factors. Interestingly, the positive association was more likely to be observed in persons with higher ApoB concentration ([116 mg/dL] for this study) than lower concentration $(99.58 \mathrm{mg} / \mathrm{dL}$ for American [7] and $106.36 \mathrm{mg} / \mathrm{dL}$ for Greece [22]), in studies with relative large sample size $([5,23,30] \&$ this study vs. small sample size [48]). Additionally, other reasons such as the differences in design of study (cross-sectional vs. prospective), different obesity (Chinese vs. Western population), and covariates that were adjusted might also provide an explanation for the inconsistent findings among these studies.

It is worth noting that $\mathrm{ApoB}$ and $\mathrm{ApoB} / \mathrm{A} 1$ ratio showed higher OR for T2D, compared with the TC, TG and LDLC. These findings were supported by previous study results. An aboriginal Canadian study indicated that plasma ApoB concentration was positively associated with T2D risk and was superior to LDL-C in predicting the disease in aboriginal Canadian population [49]. Additionally, ApoB showed a stronger association with the incident T2D compared with conventional lipid measurements in Korean populations [50]. Previous study revealed that
$\mathrm{ApoB} / \mathrm{A} 1$ ratio added significant information for predicting insulin resistance [51]. Indeed, $A$ poB was contained in each of very low LDL, intermediate-density lipoprotein, LDL-C, and lipoprotein(a) particles [8]. Taken together, these finds suggested that Apo levels either separately for ApoB or together as calculated $\mathrm{ApoB} / \mathrm{A} 1$ ratio might provide additional information for predicting the incident T2D.

\section{Mechanisms}

The association of serum ApoA1, ApoB, and ApoB/A1 ratio with T2D may be through various mechanisms. First, ApoA1 can reduce lipid binding capability, alter protein structure, and attenuate ability of catalyzing cholesterol efflux from macrophages [17]. In addition, ApoA1 is able to improve glucose tolerance via increasing glucose uptake into skeletal muscle and heart by adenosine monophosphate-activated protein kinasecomplex [52]. Second, ApoB inhibits lipolysis in adipocytes by acting as a metabolic pathway from liver to peripheral fat [53]. Dysregulation of $\mathrm{ApoB}$ metabolism can consequently cause insulin resistance [54]. Furthermore, path analyses in the present study confirmed an impact of ApoB on BMI. Third, oxidative stress and inflammation exert important roles in T2D pathophysiology. Several studies have demonstrated that ApoA1 protects mice against inflammation and oxidative stress-induced damage via clearing proinflammatory lipids and decreasing plasma malondialdehyde levels and intestinal inflammation in a Cyclooxygenase 2 total knockout and myeloid knockout/ cholatecontaining high fat diet model $[55,56]$. Finally, ApoB can aggravate inflammation by binding to enolase- 1 and releasing more inflammatory cytokines (e.g., tumor necrosis factor- $\alpha$, IL-1 $\beta$, and IL-6) [57].

\section{Strengths and limitations}

This study comprehensively assessed the association of ApoA1, ApoB, and ApoB/A1 ratio with T2D prevalence and FBG level. Importantly, to the best of our knowledge, this is the first report that analyzes the mediating effects of BMI on the association of ApoA1, ApoB, and ApoB/A1 ratio with T2D prevalence in Chinese adults. Next, the relatively large sample size allows us to be able to evaluate potential association among variables. Third, the availability of individual information (e.g., medication records and lifestyles) allows us to adjust for more potential confounders. Fourth, we further evaluated the effect of the interactions between ApoA1, ApoB, ApoB/ A1 ratio and individual information (e.g., sex, age, BMI, smoking, and drinking status) on T2D prevalence. Finally, by comparing apolipoprotein indicators, we found that $\mathrm{ApoB}$ and $\mathrm{ApoB} / \mathrm{A} 1$ ratio performed better in predicting T2D, which may provide specific guidance for large epidemiological surveys for T2D. Notwithstanding, 
the present study also has a few limitations. First, the causality was not determined due to the cross-sectional study design. However, apolipoprotein levels reached a steady-state in adults, and this state was mainly influenced by diets rather than glucose metabolism indices. Therefore, cause-and-effect association is less likely to be inverted. Second, although all subjects were recruited from urban communities in China, it had little influence on the association of ApoA1, ApoB and ApoB/A1 ratio with T2D prevalence or FBG level. Third, previous study reported dietary nutrients were related to Apo concentrations [58], however, we did not rule out the effects of dietary nutrients, which might attenuate the underlying associations. Fourth, although daily physical activity was assessed by a physical activity questionnaire, subjective recall bias and day-to-day fluctuations from daily physical activity in our study were possible, but which might have minimal impact on the present results due to daily physical activity was relative stable in participants with an average age of 60.3 years. Finally, although attempts to control for the potential confounders have been made, residual confounding may not be ruled out as reported by other observational studies.

\section{Conclusion}

In summary, this study provides support for the modulating effect of serum $A p o A 1, A p o B$, and $A p o B / A 1$ ratio on T2D prevalence and FBG level. Of these, ApoB and ApoB/A1 ratio are appropriate apolipoprotein indicators for predicting T2D in Chinese adults. Replication of these findings in large prospective studies is anticipated.

\section{Abbreviations \\ ANCOVAs: Analyses of covariance; ApoA1: Apolipoprotein A1; ApoB: Apolipoprotein B; AUC: Area under curve; BMl: Body mass index; Cls: Confidence intervals; CV: Coefficient of variation; FBG: Fasting blood glucose; HDL-C: High-density lipoprotein cholesterol; HR: Hazard ratio; IL: Interleukins; LDL-C: Low-density lipoprotein cholesterol; ORs: Odds ratios; ROC: Receiver-operating characteristic curves; SD: Standard deviation; T2D: Type 2 diabetes; TC: Total cholesterol; TG: Triglyceride}

\section{Supplementary Information}

The online version contains supplementary material available at https://doi. org/10.1186/s12902-021-00726-5.

Additional file 1: Supplementary Figure 1. The ROC curves parameters for Apo A1 level in predicting T2D. ROC, Receivers Operator Characteristic; Apo, Apolipoprotein; T2D, Type 2 diabetes.

Additional file 2: Supplementary Figure 2. The ROC curves parameters for ApoB level in predicting T2D. Abbreviations were showed in Supplementary Fig. 1.

Additional file 3: Supplementary Figure 3. The ROC curves parameters for ApoB/A1 ratio in predicting T2D. Abbreviations were showed in Supplementary Fig. 1.

Additional file 4: Supplementary Table 1. The multivariable associations of ApoA1, ApoB levels and ApoB/A1 ratio with T2D prevalence by subgroups $(\mathrm{OR}, 95 \% \mathrm{Cl})$. Supplementary Table 2 The associations of TC, TG, HDL and LDL levels with T2D prevalence (OR, 95\% Cl).

\section{Acknowledgements}

We thank all staffs and participants involved in this study.

\section{Authors' contributions}

Hongli Dong conceived and designed the research; Hongli Dong, Liang Gao, and Yaju Zhang collected the data; Liang Gao and Yaju Zhang performed the data analysis and drafted this paper, Hongli Dong and Xingmin Wang critically revised the manuscript. Xingmin Wang implemented English language editing. And Hongli Dong had primary responsibility for final content. All authors read and approved the final manuscript.

\section{Funding}

This study was partly supported by the 2020 Innovation and Entrepreneurship Program of Jiangsu Province (Doctor Funds of the Innovation and Entrepreneurship Program). This program contributed in the detection of blood biochemical parameters.

\section{Availability of data and materials}

The datasets used and/or analyzed during the current study available from the corresponding author on reasonable request.

\section{Declarations}

\section{Ethics and consent to participate}

Written informed consent was obtained from all participants. This study was conducted based on the Declaration of Helsinki and was approved by the Ethics Committee of Nantong Maternal and Child Healthcare Hospital (Y2018020).

\section{Consent for publication}

Not applicable.

\section{Competing interests}

The authors declare that they have no conflict of interest.

\section{Author details}

${ }^{1}$ Department of Clinical Laboratory, Affiliated Maternity \& Child Health Care Hospital of Nantong University, Nantong 226018, Jiangsu Province, China. ${ }^{2}$ Finance Section, Affiliated Traditional Chinese Medicine Hospital of Nantong University, Nantong 226018, Jiangsu Province, China. ${ }^{3}$ Nantong Institute of Genetics and Reproductive Medicine, Affiliated Maternity \& Child Health Care Hospital of Nantong University, Nantong 226018, Jiangsu Province, China. ${ }^{4}$ Scientific Education Section, Affiliated Maternity \& Child Health Care Hospital of Nantong University, Nantong 226018, Jiangsu Province, China.

Received: 28 September 2020 Accepted: 25 March 2021

Published online: 01 April 2021

References

1. International Diabetes Federation. IDF Diabetes Atlas. 9th edn. Brussels: International Diabetes Federation; 2019. Available from: https://www.dia betesatlas.org/en/resources/.

2. National Diabetes Research Group. Diabetes mellitus survey of 300,000 in fourteen provinces and cities of China. Chin Med J. 1981;20:678-81.

3. Yoshino G, Hirano T, Kazumi T. Atherogenic lipoproteins and diabetes mellitus. J Diabetes Complicat. 2002;16(1):29-34. https://doi.org/10.1016/S1 056-8727(01)00199-4.

4. Goldberg IJ. Clinical review 124: diabetic dyslipidemia: causes and consequences. J Clin Endocrinol Metab. 2001;86(3):965-71. https://doi.org/1 0.1210/jcem.86.3.7304

5. Seo MH, Bae JC, Park SE, Rhee EJ, Park CY, Oh KW, et al. Association of lipid and lipoprotein profiles with future development of type 2 diabetes in nondiabetic Korean subjects: a 4-year retrospective, longitudinal study. J Clin Endocrinol Metab. 2011;96:2011-1857.

6. Ley SH, Harris SB, Connelly PW, Mamakeesick M, Gittelsohn J, Wolever TM, et al. Utility of non-high-density lipoprotein cholesterol in assessing incident type 2 diabetes risk. Diabetes Obes Metab. 2012;14(9):821-5. https://doi. org/10.1111/j.1463-1326.2012.01607.x.

7. Ndumele CE, Matsushita K, Astor B, Virani SS, Mora S, Williams KE, et al. Apolipoproteins do not add prognostic information beyond lipoprotein cholesterol measures among individuals with obesity and insulin resistance 
syndromes: the ARIC study. Eur J Prev Cardiol. 2014;21 (7):866-75. https://doi. org/10.1177/2047487312465523.

8. Barter PJ, Ballantyne CM, Carmena R, Castro Cabezas M, Chapman MJ, Couture $\mathrm{P}$, et al. Apo B versus cholesterol in estimating cardiovascular risk and in guiding therapy: report of the thirty-person/ten-country panel. J Intern Med. 2006;259(3):247-58. https://doi.org/10.1111/j.1365-2796.2006.01 616.x

9. Brunzell JD, Davidson M, Furberg CD, Goldberg RB, Howard BV, Stein JH, et al. Lipoprotein management in patients with cardiometabolic risk: consensus statement from the American Diabetes Association and the American College of Cardiology Foundation. Diabetes Care. 2008;31(4):81122. https://doi.org/10.2337/dc08-9018.

10. Rader DJ, Hoeg JM, Brewer HB Jr. Quantitation of plasma apolipoproteins in the primary and secondary prevention of coronary artery disease. Ann Intern Med. 1994;120(12):1012-25. https://doi.org/10.7326/0003-4819-12 0-12-199406150-00008.

11. Sniderman AD, Faraj M. Apolipoprotein B, apolipoprotein A-I, insulin resistance and the metabolic syndrome. Curr Opin Lipidol. 2007;18(6):633-7. https://doi.org/10.1097/MOL.0b013e3282fodd33.

12. van der Vorst EPC. High-density lipoproteins and Apolipoprotein A1. Subcell Biochem. 2020;94:399-420. https://doi.org/10.1007/978-3-030-41769-7_16.

13. Andersen $L H$, Miserez AR, Ahmad Z, Andersen RL. Familial defective apolipoprotein B-100: A review. J Clin Lipidol. 2016;10(6):1297-302. https:// doi.org/10.1016/j.jacl.2016.09.009.

14. Tang S, Tabet F, Cochran BJ, Cuesta Torres LF, Wu BJ, Barter PJ, et al. Apolipoprotein A-I enhances insulin-dependent and insulin-independent glucose uptake by skeletal muscle. Sci Rep. 2019;9(1):1350. https://doi.org/1 0.1038/s41598-018-38014-3.

15. Wu BJ, Sun YD, Ong KL, Li Y, Tang SD, Barter PJ, et al. Apolipoprotein A-l protects against pregnancy-induced insulin resistance in rats. Arterioscler Thromb Vasc Biol. 2019;39(6):1160-71. https://doi.org/10.1161/ATVBAHA.11 8.312282.

16. Zhang X, Zhang W, Saraf SL, Nouraie M, Han J, Gowhari M, et al. Genetic polymorphism of APOB is associated with diabetes mellitus in sickle cell disease. Hum Genet. 2015;134(8):895-904. https://doi.org/10.1007/s00439-01 5-1572-3.

17. Domingo-Espin J, Nilsson O, Bernfur K, Del Giudice R, Lagerstedt JO. Sitespecific glycations of apolipoprotein A-I lead to differentiated functional effects on lipid-binding and on glucose metabolism. Biochim Biophys Acta Mol basis Dis. 2018;9:23.

18. Duan $Y$, Wu Z, Liu B. Relation of plasma glucose levels to serum lipids and apolipoproteins levels in middle and old age males. Hua Xi Yi Ke Da Xue Xue Bao. 2001;32(3):382-4.

19. Retnakaran R, Ye C, Connelly PW, Hanley AJ, Sermer M, Zinman B. Serum apoA1 (Apolipoprotein A-1), insulin resistance, and the risk of gestational diabetes mellitus in human pregnancy-brief report. Arterioscler Thromb Vasc Biol. 2019:39(10):2192-7. https://doi.org/10.1161/ATVBAHA.119.313195.

20. Makaridze Z, Giorgadze E, Asatiani K. Apolipoprotein B/apolipoprotein A-I ratio in relation to the metabolic syndrome, its components, total cholesterol and low-density lipoprotein cholesterol in the population of Georgia. Georgian Med News. 2014;234:32-9.

21. Zhong $L$, Li Q, Jiang Y, Cheng D, Liu Z, Wang BQ, et al. The ApoB/ApoA ratio is associated with metabolic syndrome and its components in a Chinese population. Inflammation. 2010;33(6):353-8. https://doi.org/10.1007/ s10753-010-9193-4.

22. Mellor DD, Georgousopoulou EN, D'Cunha NM, Naumovski N, Chrysohoou C, Tousoulis D, et al. Association between lipids and apolipoproteins on type 2 diabetes risk; moderating effects of gender and polymorphisms; the ATTICA study. Nutr Metab Cardiovasc Dis. 2020;30(5):788-95. https://doi. org/10.1016/j.numecd.2020.01.008.

23. Malyala R, Rapi L, Nash MM, Prasad GVR. Serum Apolipoprotein B and A1 concentrations predict late-onset Posttransplant diabetes mellitus in prevalent adult kidney transplant recipients. Can J Kidney Health Dis. 2019;6: 2054358119850536

24. Wu X, Yu Z, Su W, Isquith DA, Neradilek MB, Lu N, et al. Low levels of ApoA1 improve risk prediction of type 2 diabetes mellitus. J Clin Lipidol. 2017;11(2):362-8. https://doi.org/10.1016/j.jacl.2017.01.009.

25. Hwang YC, Ahn HY, Park SW, Park CY. Association of HDL-C and apolipoprotein A-I with the risk of type 2 diabetes in subjects with impaired fasting glucose. Eur J Endocrinol. 2014;171(1):137-42. https://doi.org/10.153 0/EJE-14-0195.
26. Hashemi M, Saadat M, Behjati M, Kelishadi R. Comparison of serum Apolipoprotein levels of diabetic children and healthy children with or without diabetic parents. Cholesterol. 2012;490381:3.

27. Onat A, Komurcu-Bayrak E, Can G, Kucukdurmaz Z, Hergenc G, ErginelUnaltuna $\mathrm{N}$, et al. Apolipoprotein $\mathrm{A}-\mathrm{I}$ positively associated with diabetes in women independently of apolipoprotein E genotype and apolipoprotein B levels. Nutrition. 2010;26(10):975-80. https://doi.org/10.1016/j.nut.2009.09. 023.

28. Chou YC, You SL, Bai CH, Liao YC, Wei CY, Sun CA, et al. Utility of apolipoprotein measurements in predicting incident type 2 diabetes: a Chinese cohort study. J Formos Med Assoc. 2020;119(1):51-8. https://doi. org/10.1016/j.jfma.2019.03.001.

29. Zheng $\mathrm{S}$, Han $\mathrm{T}$, Xu H, Zhou H, Ren X, Wu P, et al. Associations of apolipoprotein B/apolipoprotein A-I ratio with pre-diabetes and diabetes risks: a cross-sectional study in Chinese adults. BMJ Open. 2017;7:2016014038

30. Hwang YC, Ahn HY, Kim WJ, Park CY, Park SW. Increased apoB/A-I ratio independently associated with type 2 diabetes mellitus: cross-sectional study in a Korean population. Diabet Med. 2012;29(9):1165-70. https://doi. org/10.1111/j.1464-5491.2012.03622.x.

31. Pare G, Caku A, McQueen M, Anand SS, Enas E, Clarke R, et al. Lipoprotein(a) levels and the risk of myocardial infarction among 7 ethnic groups. Circulation. 2019;139(12):1472-82. https://doi.org/10.1161/CIRCULATIONA HA. 118.034311.

32. Ng M, Fleming T, Robinson M, Thomson B, Graetz N, Margono C, et al. Global, regional, and national prevalence of overweight and obesity in children and adults during 1980-2013: a systematic analyses for the global burden of disease study 2013. Lancet. 2014;384(9945):766-81. https://doi. org/10.1016/50140-6736(14)60460-8.

33. Yoon KH, Lee JH, Kim JW, Cho JH, Choi YH, Ko SH, et al. Epidemic obesity and type 2 diabetes in Asia. Lancet. 2006;368(9548):1681-8. https://doi.org/1 0.1016/S0140-6736(06)69703-1.

34. Butkowski EG, Jelinek HF. Hyperglycaemia, oxidative stress and inflammatory markers. Redox Rep. 2017;22(6):257-64. https://doi.org/10.1080/13510002.2 016.1215643.

35. Su X, Peng D. The exchangeable apolipoproteins in lipid metabolism and obesity. Clin Chim Acta. 2020;503:128-35. https://doi.org/10.1016/j.cca.2020. 01.015 .

36. Zhang C, Ho S. Validity and reproducibility of a food frequency questionnaire among Chinese women in Guangdong province. Asia Pac J Clin Nutr. 2009;18(2):240-50.

37. Dong $H L$, Tang $X Y$, Deng $Y Y$, Zhong $Q W$, Wang $C$, Zhang ZQ, et al. Urinary equol, but not daidzein and genistein, was inversely associated with the risk of type 2 diabetes in Chinese adults. Eur J Nutr. 2020;59(2):719-28. https:// doi.org/10.1007/s00394-019-01939-0.

38. Baron RM, Kenny DA. The moderator-mediator variable distinction in social psychological research: conceptual, strategic, and statistical considerations. J Pers Soc Psychol. 1986;51(6):1173-82. https://doi.org/10.1037/0022-3514.51. 6.1173.

39. Chen W, Wu Y, Lu Q, Wang S, Xing D. Endogenous ApoA-l expression in macrophages: a potential target for protection against atherosclerosis. Clin Chim Acta. 2020;505:55-9. https://doi.org/10.1016/j.cca.2020.02.025.

40. Mahley RW, Innerarity TL, Jr Rall SC, Weisgraber KH. Plasma lipoproteins: apolipoprotein structure and function. J Lipid Res. 1984;25(12):1277-94. https://doi.org/10.1016/50022-2275(20)34443-6.

41. Fruchart JC. Lipoprotein heterogeneity and its effect on apolipoprotein assays. Scand J Clin Lab Investig Suppl. 1990;198:51-7.

42. Francis MC, Frohlich JJ. Coronary artery disease in patients at low risk-apolipoprotein $\mathrm{Al}$ as an independent risk factor. Atherosclerosis. 2001;155(1): 165-70. https://doi.org/10.1016/S0021-9150(00)00528-1.

43. Walldius $\mathrm{G}$, Jungner I. Apolipoprotein A-I versus HDL cholesterol in the prediction of risk for myocardial infarction and stroke. Curr Opin Cardiol. 2007;22(4):359-67. https://doi.org/10.1097/HCO.0b013e3281bd8849.

44. Papachristou NI, Blair HC, Kypreos KE, Papachristou DJ. High-density lipoprotein (HDL) metabolism and bone mass. J Endocrinol. 2017;233(2): R95-R107. https://doi.org/10.1530/JOE-16-0657.

45. Salomaa V, Havulinna A, Saarela O, Zeller T, Jousilahti P, Jula A, et al. Thirtyone novel biomarkers as predictors for clinically incident diabetes. PLoS One. 2010;5:0010100.

46. Brahimaj A, Ligthart S, Ikram MA, Hofman A, Franco OH, Sijbrands EJG, et al. Serum levels of Apolipoproteins and incident type 2 diabetes: a prospective 
cohort study. Diabetes Care. 2017;40(3):346-51. https://doi.org/10.2337/dc1 6-1295.

47. Chen ES, Mazzotti DR, Furuya TK, Cendoroglo MS, Ramos LR, Araujo LQ, et al. Apolipoprotein A1 gene polymorphisms as risk factors for hypertension and obesity. Clin Exp Med. 2009;9(4):319-25. https://doi.org/1 0.1007/s10238-009-0051-3.

48. Lappas M, Georgiou HM, Velagic A, Willcox JC, Permezel M, Shub A. Do postpartum levels of Apolipoproteins prospectively predict the development of type 2 diabetes in women with previous gestational diabetes mellitus? Exp Clin Endocrinol Diabetes. 2019;127(6):353-8. https:// doi.org/10.1055/a-0577-7700.

49. Ley SH, Harris SB, Connelly PW, Mamakeesick M, Gittelsohn J, Wolever TM, et al. Association of apolipoprotein B with incident type 2 diabetes in an Aboriginal Canadian population. Clin Chem. 2010;56(4):666-70. https://doi. org/10.1373/dinchem.2009.136994.

50. Hwang YC, Ahn HY, Yu SH, Park SW, Park CY. Atherogenic dyslipidaemic profiles associated with the development of Type 2 diabetes: a 3.1-year longitudinal study. Diabet Med. 2014;31:24-30.

51. Sierra-Johnson J, Romero-Corral A, Somers VK, Lopez-Jimenez F, Walldius G, Hamsten A, et al. ApoB/apoA-I ratio: an independent predictor of insulin resistance in US non-diabetic subjects. Eur Heart J. 2007;28(21):2637-43. https://doi.org/10.1093/eurheartj/ehm360.

52. Fritzen AM, Domingo-Espín J, Lundsgaard AM, Kleinert M, Israelsen I, Carl CS, et al. ApoA-1 improves glucose tolerance by increasing glucose uptake into heart and skeletal muscle independently of AMPKa (2). Mol Metab. 2020:35:4.

53. Skogsberg J, Dicker A, Rydén M, Aström G, Nilsson R, Bhuiyan H, et al. ApoB100-LDL acts as a metabolic signal from liver to peripheral fat causing inhibition of lipolysis in adipocytes. PLoS One. 2008;3:20.

54. Watts GF, Ooi EM, Chan DC. Therapeutic regulation of apoB100 metabolism in insulin resistance in vivo. Pharmacol Ther. 2009;123(3):281-91. https://doi. org/10.1016/j.pharmthera.2009.04.005.

55. Meriwether D, Sulaiman D, Volpe C, Dorfman A, Grijalva V, Dorreh N, et al. Apolipoprotein A-I mimetics mitigate intestinal inflammation in COX2dependent inflammatory bowel disease model. J Clin Invest. 2019;129(9): 3670-85. https://doi.org/10.1172/JC1123700.

56. Gabás-Rivera C, Barranquero C, Martínez-Beamonte R, Navarro MA, Surra JC, Osada J. Dietary squalene increases high density lipoprotein-cholesterol and paraoxonase 1 and decreases oxidative stress in mice. PLoS One. 2014;9(8): e104224. https://doi.org/10.1371/journal.pone.0104224.

57. Lee JY, Kang MJ, Choi JY, Park JS, Park JK, Lee EY, et al. Apolipoprotein B binds to enolase-1 and aggravates inflammation in rheumatoid arthritis. Ann Rheum Dis. 2018;77(10):1480-9. https://doi.org/10.1136/annrheumdis-2 018-213444.

58. Mente A, Dehghan M, Rangarajan S, McQueen M, Dagenais G, Wielgosz A, et al. Association of dietary nutrients with blood lipids and blood pressure in 18 countries: a cross-sectional analysis from the PURE study. Lancet Diabetes Endocrinol. 2017;5(10):774-87. https://doi.org/10.1016/S22138587(17)30283-8.

\section{Publisher's Note}

Springer Nature remains neutral with regard to jurisdictional claims in published maps and institutional affiliations.

Ready to submit your research? Choose BMC and benefit from:

- fast, convenient online submission

- thorough peer review by experienced researchers in your field

- rapid publication on acceptance

- support for research data, including large and complex data types

- gold Open Access which fosters wider collaboration and increased citations

- maximum visibility for your research: over $100 \mathrm{M}$ website views per year

At BMC, research is always in progress.

Learn more biomedcentral.com/submissions 